\title{
Structure of Gaseous Detonation Waves and Chemical Kinetics
}

\author{
P.J. Van Tiggelen \\ Laboratoire de Physico-Chimie de la Combustion, Université Catholique de Louvain, Louvain-la-Neuve, \\ Belgique
}

\begin{abstract}
The tridimensional unsteady character of the flow field for detonations has been established about thirty years ago. The shock pattern is rather complex and the complete description of the phenomenon remains beyond the power of fast computer even today. However the classical thermodynamic approach allows to grasp what are the dominating factors of the whole process: leading shock inducing an exothermic reaction governed by the chemical kinetic mechanism of the heat release. The dynamics of gaseous detonation is coupled therefore closely to the rate of the heat release which in turn influences the time-dependent shock structure of the front.

Measurements of the detonation velocity (D) are close to the values of the Chapman-Jouguet model of a detonation which is based on full thermodynamic equilibrium. But, the structure as evidenced from the soot records is more sensitive to the initial conditions : pressure, equivalence ratio, diluent nature and percentage, as well as the type of fuels, promoters and inhibitors of combustion processes. From systematic measurements of the length (L) of the detonation cell imprinted on soot for several mixtures, it has been possible to demonstrate the promoting role of hydrogen, and the inhibiting role of halocarbons on the detonation of $\mathrm{CO} / \mathrm{O} 2 / \mathrm{Ar}$ mixtures. Some detailed studies of $\mathrm{OH}$ emission, shock velocity of the unsteady leading shock, pressure inside a cell have shown the self-similar character of the flow field inside one cell. It demonstrates that the detonation phenomenon can be viewed as a periodic reinitiation of reactive shocks at a frequency identical to the reciprocal of the characteristic time as defined by the ratio: $\mathrm{L} / \mathrm{D}$. Irregular cell structure can occur in more complex mixtures such as $\mathrm{CH} 4 / \mathrm{O} 2$. In that case the higher values of the activation energy of the conversion process induce a much higher sensitivity to the elaborate temperature fluctuations of the flow field. More recently,numerical simulations have allowed to model the unsteady bidimensional model of a detonation. The following papers could be referred to for more details about this approach of the gaseous detonations:

-J.C.LIBOUTON, M.DORMAL, and P.J.VAN TIGGELEN, Progress in Astronautics and Aeronautics, Vol.75 pp 358-369 (1981)

-P.J.VAN TIGGELEN et J.C.LIBOUTON, Annales de Physique ,Vol.14 pp 649-660 (1989) -M.H.LEFEBVRE, E.S.ORAN, K.KAILASANATH,and P.J.VAN TIGGELEN , Progress in Astronautics and Aeronautics, Vol. 153 pp 64-76 (1992)

-M.H.LEFEBVRE, E.S.ORAN, K.KAILASANATH, and P.J.VAN TIGGELEN, Combustion and Flame Vol. 95 pp 206-218 (1993)
\end{abstract}

